\title{
Mathematical Modelling of Spatiotemporal Dynamics of Oxygen in a Plankton System
}

\author{
Y. Sekerci, S. Petrovskii *
}

Department of Mathematics, University of Leicester,University Road, Leicester LE1 7RH, U.K.

\begin{abstract}
Oxygen production due to phytoplankton photosynthesis is a crucial phenomenon underlying the dynamics of marine ecosystems. However, most of the existing literature focus on other aspects of the plankton community functioning, thus leaving the issue of the coupled oxygen-plankton dynamics understudied. In this paper, we consider a generic model of the oxygen-phytoplankton-zooplankton dynamics to make an insight into the basic properties of the plankton-oxygen interactions. The model is analyzed both analytically and numerically. We first consider the nonspatial model and show that it predicts possible oxygen depletion under certain environmental conditions. We then consider the spatially explicit model and show that it exhibits a rich variety of spatiotemporal patterns including travelling fronts of oxygen depletion, dynamical stabilization of unstable equilibrium and spatiotemporal chaos.
\end{abstract}

Keywords and phrases: plankton dynamics, photosynthesis, oxygen, pattern formation

Mathematics Subject Classification: 92D40, 35B36, 35Q92, 37N25

\section{Introduction}

About 70 per cent of the earth's surface is covered by the oceans [13]. Plankton are floating organisms of many different phyla living in the pelagial of the oceans. Their functional classification is based on trophic level, size, and distribution. Autotrophs, i.e., primary producers, constitute phytoplankton, whereas heterotrophs, i.e., consumers, include bacterioplankton and zooplankton. Like terrestrial plants, phytoplankton contain chlorophyll. Correspondingly, phytoplankton consists of photosynthetic organisms, usually single celled, and they are responsible for the oxygen production in the world's oceans. Phytoplankton is estimated to be responsible for at least one half of the total photosynthetic activity on Earth, thereby accounting considerably for the production of the atmospheric oxygen $[11,26]$.

Dynamics of oxygen production due to phytoplankton photosynthetic activity has long been a focus of research, both in field measurements and in laboratory. The earliest measurement of oxygen production (dated back to almost 90 years ago) was made by determining changes in oxygen concentration in bottles submerged in the water by using Winkler techniques [9]. In his classical book, Harris [11] considered the connection between growth rate of oxygen production and photosynthesis, as photosynthesis constitutes the main source of energy accessible to the cells. Paasche [28] showed that the concentration of oxygen in the ocean is due to the photosynthetic activity of phytoplankton, which is mainly responsible for the

\footnotetext{
${ }^{*}$ Corresponding author. E-mail: sp237@le.ac.uk
} 
photosynthetic process in ocean. Phytoplankton therefore play an essential role in maintaining life on Earth in terms of oxygen production [26] but it also responsible for food production in marine ecosystem [5]. Indeed, the aquatic food chain is based on the plankton community.

Note that, whilst phytoplankton is a major oxygen producer, both phyto- and zooplankton as well as macrophytes are also oxygen consumers [23], in particular through the decomposition process of marine sediment in bottom of the ocean. The importance of plankton decomposition process in relation to oxygen balance in aquatic ecosystems is discussed in [41]. In addition to what is happening in the pelagic zone, marine sediments are formed by dead algae and macrophytes. These particles sink to the bottom of the ocean and are exposed to a biochemical process. Large amounts of dissolved oxygen are utilized by these biochemical processes $[7,12,33,36,39]$. Sediment decomposition process needs oxygen and dissolved oxygen is utilized for this process. This, in turn, can cause the ocean's dissolved oxygen concentration to become low.

Along with the experimental studies, mathematical modelling has been recognized as a powerful theoretical tool to study plankton dynamics. There is considerable literature concerned with various aspects of plankton dynamics in space and time. Conceptual prey-predator-type models to describe the phytoplankton and zooplankton interaction in marine ecosystems subject to turbulent mixing were considered in much detail in $[4,16,20,21,31]$ but with no attention to the oxygen production. In another mathematical study, Edwards and Brindley [7] investigated the dynamics of a coupled plankton-nutrients system, but did not pay any attention to their possible relation to dissolved oxygen. There are relatively few papers where oxygen production is considered explicitly $[2,22-24]$ but these papers leave out of the scope some important features of plankton dynamics such as, for instance, the pronounced heterogeneity of its spatial distribution (known as plankton patchiness). In particular, Marchettini et al. [22] studied the tropic dynamics by developing a mathematical model of biochemical processes in a lagoon ecosystem. They considered the dissolved oxygen concentration in a multi-component system. In another modelling study, Allegretto et al. [2] showed the existence of periodic solutions in Italian coastal lagoons. A nonlinear model accounting for the interplay between the physical and chemical processes in coastal ecosystems was considered by Mocenni [24]; however, their study did not address relation between the plankton population and oxygen concentration. An algae-oxygen model has been proposed and analyzed by Misra [23] subject to the effect of only 'exogenous' factors (such as light, wind intensity, temperature, phosphorus, eutrophication, etc), hence leaving the internal plankton-oxygen dynamics out of the focus.

We therefore observe that, in spite of a vast literature related to the marine ecosystems modelling, the dynamics of the dissolved oxygen concentration as an essential component of a plankton system has not been studied in sufficient detail. Meanwhile, this issue is obviously of significant practical and theoretical importance. Correspondingly, the aim of this paper is to consider the effect of the phyto-zooplankton (prey-predator) interactions on the dynamics of the dissolved oxygen. In its turn, this requires a good understanding of the properties of the baseline two-component oxygen-phytoplankton system.

In view of the above, the paper is structured as follows. In the next section, a new mathematical model of oxygen-phytoplankton dynamics is proposed and analyzed. In Section 3, we extend our model to include the zooplankton. In Sections 4 and 5, the properties of the three-component system are studied by extensive numerical simulations both in spatial and nonspatial models to reveal rich spatiotemporal dynamics including chaos and travelling fronts of extinction. In Section 6, the ecological relevance and potential importance of our findings are discussed.

\section{The baseline model}

We begin with a simple conceptual model that only takes into account the temporal dynamics of the oxygen itself and the phytoplankton as its main producer:

$$
\frac{d c(t)}{d t}=A f(c) u-m c
$$




$$
\frac{d u(t)}{d t}=g(c, u) u
$$

Here $c$ and $u$ are the concentration of the dissolved oxygen and the phytoplankton density, respectively, at time $t, f$ is the amount of oxygen produced per unit time and per unit phytoplankton mass, $g$ is the per capita phytoplankton growth rate, $A$ is a coefficient that can take into account the effect of relevant environmental factors, and the term $m c$ takes into account oxygen losses, e.g. due to its diffusion to the atmosphere, plankton breathing, etc. Note that Eq. (2.1) is linear with respect to $u$, and indeed we are not aware about any evidence that the photosynthesis rate can depend on phytoplankton density. On the contrary, Eq. (2.2) should normally be nonlinear with respect to $u$ (hence the dependence of $g$ on $u$ ) as the high phytoplankton density is known to damp its growth, e.g. due to self shading and/or nutrient depletion.

In order to understand what can be the properties of functions $f$ and $g$, we have to look more closely at the oxygen production and consumption. Consider $f(c)$ first. Oxygen is produced inside phytoplankton cells in photosynthesis and then diffuse through the cell membrane into the surrounding water. Diffusion flux always directed from areas with higher concentration of the diffusing substance to the areas with lower ones; the larger is the difference between the concentrations, the larger is the flux (cf. the Fick law). Therefore, for the same rate of photosynthesis, the amount of oxygen that gets through the cell membrane will be the larger the lower is the oxygen concentration in the surrounding water. Therefore, $\mathrm{f}$ should be a monotonously decreasing function of $c$. We further assume that the oxygen flux through the cell membrane tends to zero when the oxygen concentration in the water is very large, i.e., in physical terms, is close to its saturating value. The above features are qualitatively taken into account by the following parametrization:

$$
f(c)=1-\frac{c}{c+c_{0}},
$$

where $c_{0}$ is thus the half-saturation constant.

Considering phytoplankton multiplication, we assume that $g(c)=\alpha(c)-\gamma u$ where the first term describes the phytoplankton linear growth and the second term account for intraspecific competition for resources. Eq. (2.2) for the phytoplankton growth is therefore essentially the logistic growth equation where $1 / \gamma$ plays the role of the carrying capacity, which we assume does not depend on $c$. However, the linear growth rate $\alpha$ should depends on $c$, which can be seem from the following argument. Phytoplankton produce oxygen in photosynthesis during the daytime, but it needs oxygen for breathing during the night; therefore, a low oxygen concentration is unfavorable for phytoplankton and is likely to depress its reproduction. On the other hand, a phytoplankton cell cannot take more oxygen than it needs. Hence $\alpha$ should be monotonously increasing function of $c$ tending to a constant value for $c \rightarrow \infty$. The simplest parametrization for $\alpha$ is then given by the Monod function, so that for $g(c, u)$ we obtain:

$$
g(c, u)=\frac{B c}{c+c_{1}}-\gamma u,
$$

where $c_{1}$ is the half-saturation constant and $B$ is the phytoplankton maximum per capita growth rate.

With (2.3-2.4), Eqs. (2.1-2.2) take the following form:

$$
\begin{aligned}
& \frac{d c}{d t}=A\left(1-\frac{c}{c+c_{0}}\right) u-m c, \\
& \frac{d u}{d t}=\left(\frac{B c}{c+c_{1}}-\gamma u\right) u .
\end{aligned}
$$

The system (2.5-2.6) contains six parameters; however, their number can be reduced by choosing dimensionless variables as follows:

$$
t^{\prime}=t m, \quad c^{\prime}=\frac{c}{c_{0}}, \quad u^{\prime}=\frac{\gamma u}{m},
$$


and the new parameters accordingly as

$$
\hat{B}=\frac{B}{m}, \quad \hat{A}=\frac{A}{c_{0} \gamma} \quad \text { and } \quad \hat{c}=\frac{c_{1}}{c_{0}} .
$$

For convenience, we now simplify the notations by omitting the primes and hats, i.e. by changing $t^{\prime} \rightarrow t, c^{\prime} \rightarrow c, u^{\prime} \rightarrow u, \hat{A} \rightarrow A$ and $\hat{B} \rightarrow B$ and $\hat{c} \rightarrow c_{1}$. Eqs. (2.5-2.6) then take the following form:

$$
\begin{aligned}
& \frac{d c}{d t}=A\left(1-\frac{c}{c+1}\right) u-c \equiv f(c, u), \\
& \frac{d u}{d t}=\left(\frac{B c}{c+c_{1}}-u\right) u \equiv g(c, u),
\end{aligned}
$$

where all variables and parameters are now dimensionless.

The next step is to reveal the existence of the equilibria (steady states), as given by the non-negative solutions of the following system:

$$
f(c, u)=0, \quad g(c, u)=0 .
$$

Biologically meaningful system equilibria are non-negative intersection points of oxygen zero-growth isocline and phytoplankton zero-growth isocline. The shape of these (null-)isoclines is shown in Fig. 1. The first isocline (i.e. for oxygen growth) is given by the curve $\tilde{u}=\frac{\tilde{c}}{A}(\tilde{c}+1)$ and the second isocline (i.e. for phytoplankton growth) is given by $\tilde{u}=0$ and $\tilde{c}=\frac{\tilde{u} \tilde{c}_{1}}{B-\tilde{u}}$. Correspondingly, Eqs. (2.7) and (2.8) have at most two non-negative solutions. One is extinction state $(0,0)$ and the other is coexistence state $(\tilde{c}, \tilde{u})$. The extinction state exists for all parameters values. As for the coexistence state, as we will show it below, it only exists under certain conditions.

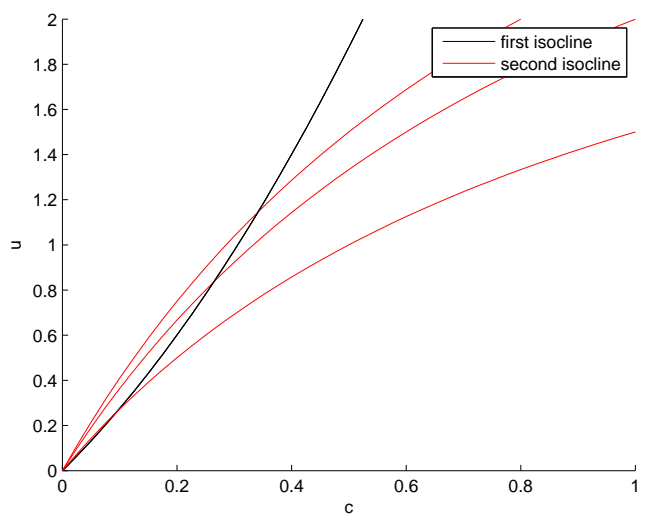

FiguRE 1. The (null-)isoclines of the oxygen-phytoplankton system (2.7-2.8). Black curve shows the oxygen isocline for $A=0.4$ and $c_{1}=1$; red curves show the phytoplankton isocline for $B=3, B=4$ and $B=4.5$, from bottom to top.

The steady state values $\tilde{c}$ and $\tilde{u}$ are the solutions of the following system:

$$
\begin{gathered}
A\left(1-\frac{\tilde{c}}{\tilde{c}+1}\right) \tilde{u}-\tilde{c}=0, \\
\left(\frac{B \tilde{c}}{\tilde{c}+c_{1}}-\tilde{u}\right) \tilde{u}=0,
\end{gathered}
$$


from which the equations for the isoclines can be obtained, respectively, as

$$
\tilde{u}=\frac{\tilde{c}}{A}(\tilde{c}+1)
$$

and

$$
\tilde{u}=\frac{B \tilde{c}}{\tilde{c}+c_{1}} .
$$

The corresponding curves are shown in Fig. 1.

Note that Eq. (2.12) can be resolved with regard to $\tilde{c}$ as follows:

$$
\tilde{c}=\frac{\tilde{u} c_{1}}{B-\tilde{u}} .
$$

Substituting (2.13) in equation (2.11), the following quadratic equation is obtained for $\tilde{u}$ :

$$
A \tilde{u}^{2}+\left(-2 A B-c_{1}^{2}+c_{1}\right) \tilde{u}+A B^{2}-c_{1} B=0,
$$

so that its solution is given by

$$
\tilde{u}=\frac{-\sigma \pm \sqrt{\sigma^{2}-4 A \kappa}}{2 A} \quad \text { and } \quad \tilde{c}=\frac{\tilde{u} c_{1}}{B-\tilde{u}}
$$

where

$$
\sigma=-2 A B-c_{1}^{2}+c_{1} \text { and } \kappa=A B^{2}-c_{1} B .
$$

It is readily seen that a unique positive root exists if the following conditions are satisfied. Consider

$$
\tilde{u}=\frac{-\sigma-\sqrt{\sigma^{2}-4 A \kappa}}{2 A}>0,
$$

then

$$
\sigma^{2}-4 A \kappa \geq 0, \quad \sigma<0,
$$

and

$$
-\sigma-\sqrt{\sigma^{2}-4 A \kappa}>0,
$$

so that

$$
0>-4 A \kappa \quad \Longrightarrow \quad \kappa>0 \quad \Longrightarrow \quad A B>c_{1} \text {. }
$$

Therefore,

$$
\tilde{u}=\frac{-\sigma-\sqrt{\sigma^{2}-4 A \kappa}}{2 A}>0 \text { for } A B>c_{1} .
$$

Thus, using the expression (2.17) for $\tilde{u}$, we have arrived to condition (2.20). We mention here that the choice of the other root instead of (2.17) results in $\tilde{u}<0$ and hence it is ecologically meaningless.

Note that the shape of the second isocline, as given by Eq. (2.12) or (2.13), depends on parameter $B$ and that taking sufficiently small values of $B$ eventually results in the disappearance of the positive steady state (see the succession of red curves in Fig. 1) so that the only remaining equilibrium is extinction. From the geometric argument, it is readily seen that the positive steady state exists if and only if the slope of the oxygen isocline at the origin is less than the slope of the phytoplankton isocline. From Eqs. (2.11) and (2.12), we obtain that the isoclines' slope is $1 / A$ and $B / c_{1}$, respectively. Correspondingly, the condition of their intersection is

$$
\frac{1}{A}<\frac{B}{c_{1}}, \quad \text { so that } \quad A B>c_{1},
$$

which obviously coincides with (2.20). We mention here that condition $A B>c_{1}$ has a clear biological interpretation. Recall that $A$ quantifies the rate of oxygen production. Therefore, condition (2.21) (or 
(2.20)) means that, since oxygen is a vital resource, its production must be high enough in order to support the phytoplankton existence.

As for the stability of the steady states, it is straightforward to see (e.g. by considering the direction of the flow in different regions of the phase plane) that the coexistence state $(\tilde{c}, \tilde{u})$ is stable for all parameter values when it is feasible, i.e. is situated in the first quarter of the plane. In that case, the extinction state $(0,0)$ is unstable and hence $(\tilde{c}, \tilde{u})$ is the global attractor. When the coexistence state is not feasible, then the extinction state is stable and acts as the global attractor.

\section{The 'advanced' three-component model}

The baseline oxygen-phytoplankton model from the previous section can be made biologically more realistic by taking into account zooplankton. Indeed, zooplankton is the main consumer of phytoplankton and it is well known that its effect can change the system's properties significantly, usually making the dynamics more complex, cf. $[7,8,18]$. In the model below, we assume that the phyto-zooplankton trophic interaction is described by the standard prey-predator model with the functional response of Holling type II. The corresponding model is described by the following differential equations:

$$
\begin{aligned}
& \frac{d c}{d t}=A\left(1-\frac{c}{c+1}\right) u-c \equiv f(c, u, v), \\
& \frac{d u}{d t}=\left(\frac{B c}{c+c_{1}}-u\right) u-\frac{u v}{u+h} \equiv g(c, u, v), \\
& \frac{d v}{d t}=\left(\frac{\beta u v}{u+h}\right)-\mu v \equiv l(c, u, v),
\end{aligned}
$$

(in dimensionless variables) where $v$ is the zooplankton density. The third term of Eq. (3.2) is the grazing of zooplankton on phytoplankton, where the coefficient $h$ is the half saturation density. The first term of Eq. (3.3) represents the growth rate of zooplankton due to predation where $\beta$ is the (dimensionless) maximum per capita growth rate of zooplankton and $\mu$ is the natural mortality. Note that, for the sake of simplicity, in Eq. (3.1) we have neglected the oxygen consumption due to the zooplankton breathing, hence assuming that the zooplankton density is not very high.

\subsection{Equilibrium analysis}

The nonspatial three-component system (3.1-3.3) has at most three equilibria.

1. The trivial equilibrium $E_{1}=(0,0,0)$ corresponding to extinction. It is readily seen that this equilibrium exists always, regardless what the parameter values are.

2. The semi-trivial equilibrium $E_{2}=(\tilde{c}, \tilde{u}, 0)$. Once $v=0$, the system $(3.1-3.3)$ is reduced to the oxygen-phytoplankton system (2.7-2.8); therefore, the results obtained in Section 2 readily apply. In particular, the steady state values $\tilde{c}$ and $\tilde{u}$ are given by Eqs. (2.11-2.13), and the condition of the equilibrium existence is given by the related condition Eq. (2.20).

3. The positive (coexistence) equilibrium $E_{3}=(\bar{c}, \bar{u}, \bar{v})$. This equilibrium exits under certain conditions that are obtained below.

The steady state values $\bar{c}, \bar{u}$ and $\bar{v}$ are the solutions of the following system:

$$
\begin{gathered}
A\left(1-\frac{\bar{c}}{\bar{c}+1}\right) \bar{u}-\bar{c}=0, \\
\left(\frac{B \bar{c}}{\bar{c}+c_{1}}-\bar{u}\right) \bar{u}-\frac{\bar{u} \bar{v}}{\bar{u}+h}=0, \\
\frac{\beta \bar{u} \bar{v}}{\bar{u}+h}-\mu \bar{v}=0,
\end{gathered}
$$


that can be solved semi-explicitly as follows:

$$
\bar{u}=\frac{\mu h}{\beta-\mu}, \quad \bar{c}=\frac{-1+\sqrt{1+4 A \bar{u}}}{2}, \quad \bar{v}=(\bar{u}+h)\left(\frac{B \bar{c}}{\bar{c}+c_{1}}-\bar{u}\right)
$$

(where we have omitted the second root for $\bar{c}$ because it is always negative and hence biologically meaningless).

It is then readily seen that all the steady state values (3.7) are positive under the following conditions:

$$
\beta>\mu, \quad \sqrt{1+4 A \bar{u}}>1 \quad \text { and } \quad \frac{B \bar{c}}{\bar{c}+c_{1}}-\bar{u}>0,
$$

which thus gives the conditions of $E_{3}$ existence. Since all the parameters in (3.8) are positive due to their biological meaning, the second condition in (3.8) holds identically; however, the first and third ones impose nontrivial restrictions on the range of biologically meaningful parameter values.

\subsection{Stability analysis}

Now we are going to discuss the stability of the steady states $E_{1}, E_{2}$ and $E_{3}$. For this reason, we calculate the Jacobian matrix of the system (3.4)-(3.6):

$$
A=\left(\begin{array}{ccc}
-\frac{A u}{(1+c)^{2}}-1 ; & A\left(1-\frac{c}{1+c}\right) ; & 0 ; \\
\frac{B c_{1} u}{\left(c+c_{1}\right)^{2}} ; & \frac{B c}{c+c_{1}}-2 u-\frac{v h}{(u+h)^{2}} ; & -\frac{u}{u+h} ; \\
0 ; & \frac{\beta v h}{(u+h)^{2}} ; & \frac{\beta u}{u+h}-\mu ;
\end{array}\right) .
$$

For each of the steady states, the eigenvalues are the solutions of the characteristic equation:

$$
\operatorname{det}\left(A_{i}-\lambda I\right)=0,
$$

where $I$ is the unit matrix and $A_{i}$ is the matrix (3.9) with the elements calculated at the steady state $E_{i}, i=1,2,3$. Below we give a brief summary of the results, details of the calculations can be found in Appendix.

- Extinction state $E_{1}$

The eigenvalues of matrix $A_{1}$ are $-\mu,-1$ and 0 . The fact that one of the eigenvalues is zero tells that the linear stability analysis is not informative. Generally speaking, in this case, one has to perform a higher order stability analysis to determine the stability of the equilibrium, e.g. by applying the HartmanGrobman theorem [10,15]. Alternatively, however, in order to avoid tedious analytical calculations, we can check the stability by numerical simulations.

- Zooplankton-free, oxygen-phytoplankton state $E_{2}$

The eigenvalues of matrix $A_{2}$ are the solutions of the following characteristic equation:

$$
\left[\left(-\frac{A \tilde{u}}{(1+\tilde{c})^{2}}-1-\lambda\right)\left(\frac{B \tilde{c}}{\tilde{c}+c_{1}}-2 \tilde{u}-\lambda\right)-\frac{A}{1+\tilde{c}} \frac{B c_{1} \tilde{u}}{\left(\tilde{c}+c_{1}\right)^{2}}\right]\left(\frac{\beta \tilde{u}}{\tilde{u}+h}-\mu-\lambda\right)=0
$$

where $\tilde{c}$ and $\tilde{u}$ are given by Eqs. (2.13) and (2.17).

The analytical solution of Eq. (3.11) is bulky and hence we do not show it here for the sake of brevity (but see Appendix for more details). Figure 2 shows each of the eigenvalues $\lambda_{1}, \lambda_{2}$ and $\lambda_{3}$ as a function on the controlling parameter $A$ for two hypothetical values $c_{1}$. It is readily seen that equilibrium $E_{2}$ can be stable or unstable. For example, for $c_{1}=0.4, E_{2}$ is stable for $A=0.42$ but it is a saddle point for $A=0.5$. 


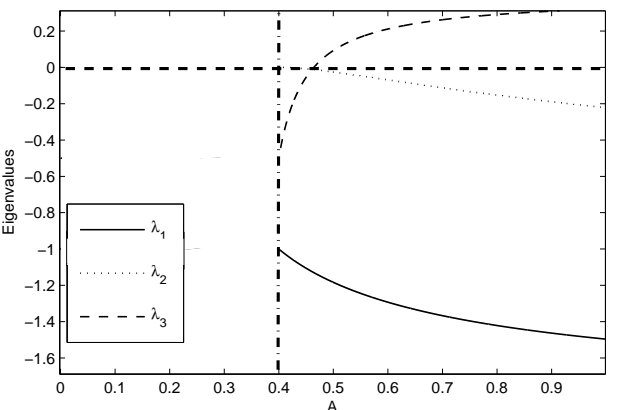

(a)

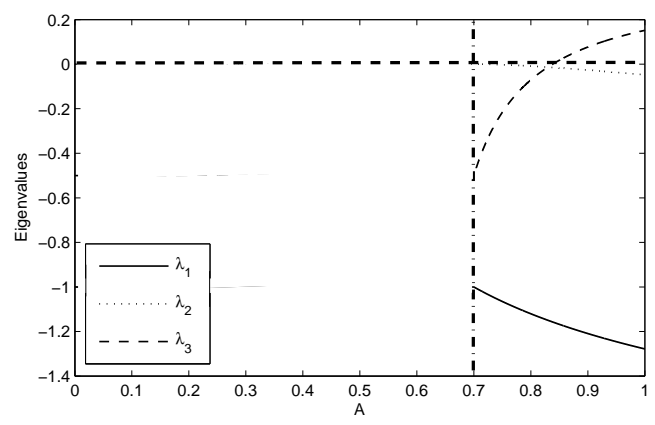

(b)

FiguRE 2. Bifurcation diagram for the zooplankton-free state $E_{2}$ for (a) $c_{1}=0.4$ and (b) $c_{1}=0.7$. The vertical line shows the feasibility condition, for the values of $A$ on the left of the line $E_{2}$ does not exist.

- Oxygen-phyto-zooplankton coexistence state $E_{3}$

For this equilibrium, the eigenvalues of the corresponding matrix $A_{3}$ are the solutions of the following characteristic equation:

$$
\begin{array}{r}
\left(\frac{\beta \bar{u}}{\bar{u}+h}-\mu-\lambda\right)\left[\left(-\frac{A \bar{u}}{(1+\bar{c})^{2}}-1-\lambda\right)\left(\frac{B \bar{c}}{\bar{c}+c_{1}}-2 \bar{u}-\frac{\bar{v} h}{(\bar{u}+h)^{2}}-\lambda\right)\right. \\
\left.-\frac{A}{1+\bar{c}}\left(\frac{B c_{1} \bar{u}}{\left(\bar{c}+c_{1}\right)^{2}}\right)\right]-\frac{\beta \bar{u} \bar{v} h}{(\bar{u}+h)^{3}}\left(\frac{A \bar{u}}{(1+\bar{c})^{2}}+1+\lambda\right)=0 .
\end{array}
$$

The analytical solution of Eq. (3.12) is extremely bulky; see Appendix for details. Figure 3 shows $\operatorname{Re} \lambda$ for each of the eigenvalues $\lambda_{1}, \lambda_{2}$ and $\lambda_{3}$ as a function on the controlling parameter $A$ for two hypothetical values $c_{1}$. Note that in this case two of the eigenvalues appear to be complex-conjugate, so that $\operatorname{Re} \lambda_{2}=\operatorname{Re} \lambda_{3}$. Of the two different real parts, $\lambda_{1}$ is distinctly negative (see the solid curve in Fig. 3) whilst $\operatorname{Re} \lambda_{2}=\operatorname{Re} \lambda_{3}$ is very small (for the given parameter set), positive for $c_{1}=0.4$ (Fig. 3a) and negative for $c_{1}=0.7$ (Fig. $3 \mathrm{~b}$ ).

\section{Numerical simulations I: temporal dynamics}

In this section, we perform numerical simulations of oxygen-phytoplankton-zooplankton nonspatial system (3.1-3.3). Understanding of the temporal dynamics creates a convenient framework for the understanding of the complex dynamics of the spatio-temporal system. In all our numerical simulations shown in this section, we fix parameters at some hypothetical values as $\beta=1, \mu=0.5$ and $h=0.1$, and vary $A$ and $c_{1}$ in a certain range. Our particular interest is to the effect of changes in parameter $A$ as it may, in term of the real-world plankton system, account for the effect of environmental changes.

Fig. 4 shows the oxygen concentration and the phyto- and zooplankton densities versus time obtained for $c_{1}=0.8$ and two different values of $A$. In the case of $A=0.3$ (Fig. 4a), all components go extinct in the large-time limit. This is not surprising as it is readily seen that, for these parameter values, the conditions (2.20) and (3.8) do not hold and hence only the extinction steady state exists. However, for $A=1$ (Fig. 4b), conditions (3.8) for the coexistence state existence hold (but the conditions (2.20) do not) so that, in the large-time limit, the densities converge to some positive steady state values (although $\bar{v}$ appears to be quite small in this case). 


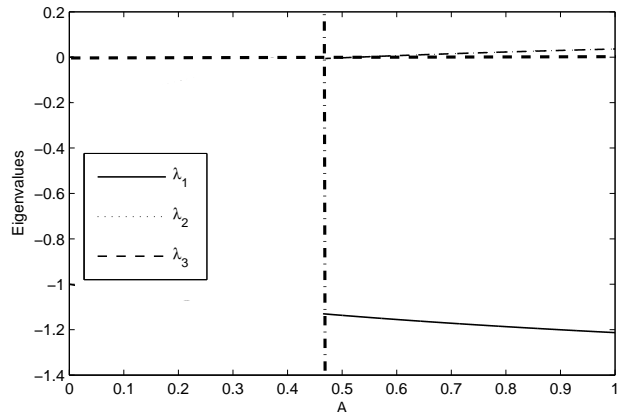

(a)

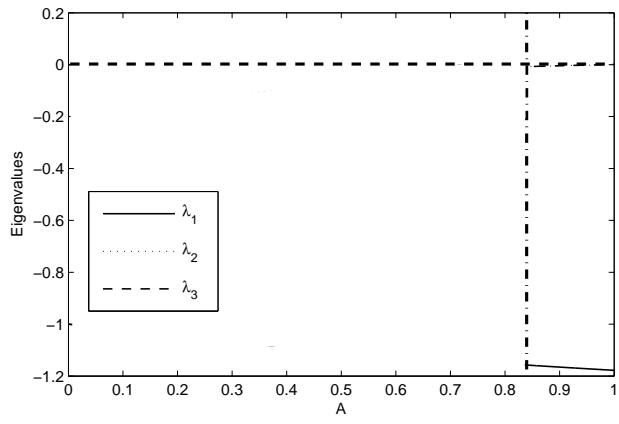

(b)

Figure 3. Bifurcation diagram for the oxygen-phyto-zooplankton coexistence state $E_{3}$ for (a) $c_{1}=0.4$ and (b) $c_{1}=0.7$. The vertical line shows the feasibility condition, for the values of $A$ on the left of the line $E_{2}$ does not exist.

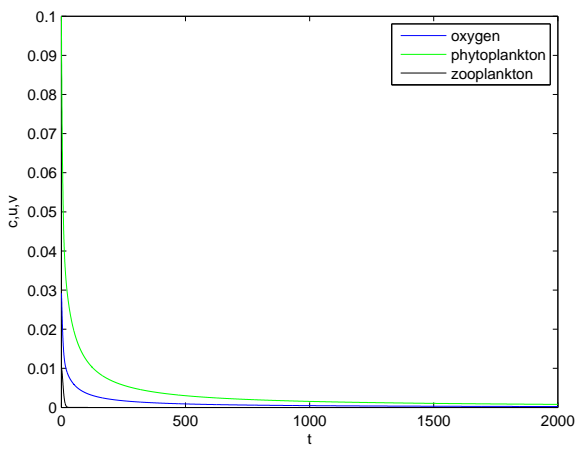

(a)

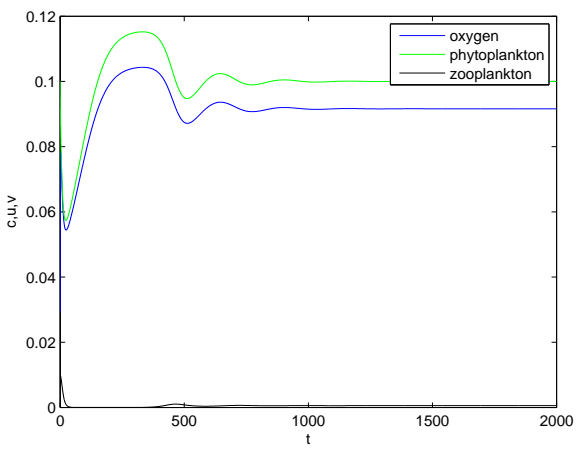

(b)

FiguRE 4 . Effect of changes in parameter $A$. The density of oxygen, phytoplankton and zooplankton against time obtained for parameter values (a) $A=0.3, c_{1}=0.8$ and (b) $A=1, c_{1}=0.8$. The initial conditions are $c_{0}=0.0292, u_{0}=0.1, v_{0}=0.01$. Other parameters are given in the text.

These simple results have important biological meaning. Once the oxygen production rate (as quantified by parameter $A$ ) becomes low, e.g. as a result of environmental changes, the available amount of oxygen may be not sufficient to support life of the plankton community, which results is the plankton extinction.

On a more technical note, we mention here that not only the extinction/persistence issue but also the rate of convergence can differ greatly for different parameter values. Figure 5 shows the simulation results obtained for $A=1$ and $c_{1}=0.1$. Obviously, all the system component go extinct in the course of time. However, we notice that, whilst for the parameters of Fig. 4 the convergence occurs over the time scale of $10^{3}$, for the parameters of Fig. 5 the convergence occurs 100 times faster.

Apart from the existence/extinction change in the system behavior, the effect of changes in parameter $A$ may have a somewhat more subtle effect on the stability of the system. Fig. 6 shows the oxygen concentration and the phyto- zooplankton densities versus time obtained for the same value of $c_{1}=0.7$ and two different values of $A$. For $A=0.9$ (Fig. 6a), the densities eventually converge to the steady state values after a sequence of damping oscillations, which obviously corresponds to $E_{3}$ being a stable focus. 


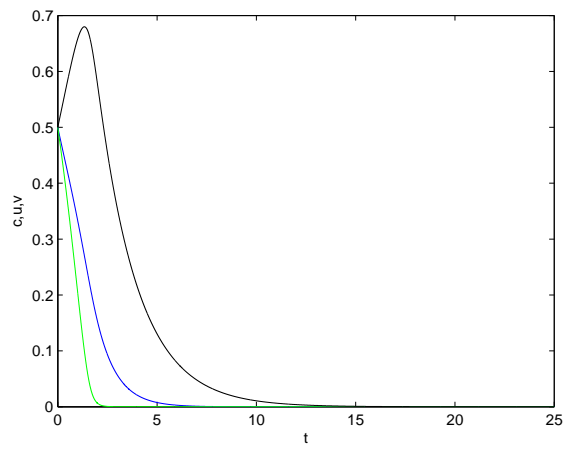

FiguRE 5. Oxygen, phyto- and zooplankton densities over time simulated for parameters $A=1$, and $c_{1}=0.1$, other parameters are the same as in the previous figure. The initial conditions are $c_{0}=0.5, u_{0}=0.5$ and $v_{0}=0.5$.

However, the situation is different for $A=1$ (Fig. 6b) where the system eventually develops periodic oscillations. This change in the system's properties is in full agreement with our analysis of the steady state stability undertaken in Section 3.1 (see also Appendix); indeed, the Hopf bifurcation occur when $A$ changes from 0.9 to 1 .

An interesting succession of dynamical regimes observed for a sequence of increasing values of $A$ is shown in Fig. 7. Figure 7a obtained for $A=0.41$ shows that oxygen and plankton densities converge to the zooplankton-free steady state $E_{2}$. Note that the initial zooplankton density drops very fast (so that the corresponding curve in Fig. 7a at this resolution almost coincides with the vertical axis).

For $A=0.5$ (Fig. $7 \mathrm{~b}$ ), the system dynamics follows a long-living transient. Over the first stage of the dynamics (up to $t \approx 1000$ ), the densities apparently converge to the zooplankton-free steady state. However, this state appears to be a saddle rather than a stable equilibrium. After staying in its vicinity for a considerable time (roughly, between $t=400$ and $t=1000$ ), the trajectory then shoots away to the vicinity of $E_{3}$ (which, for these parameter values, is a stable focus) so that the densities eventually converge to their steady state values $\bar{c}, \bar{u}$ and $\bar{v}$.

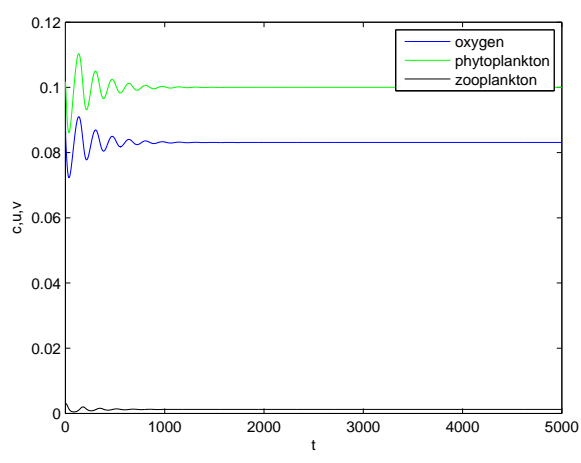

(a)

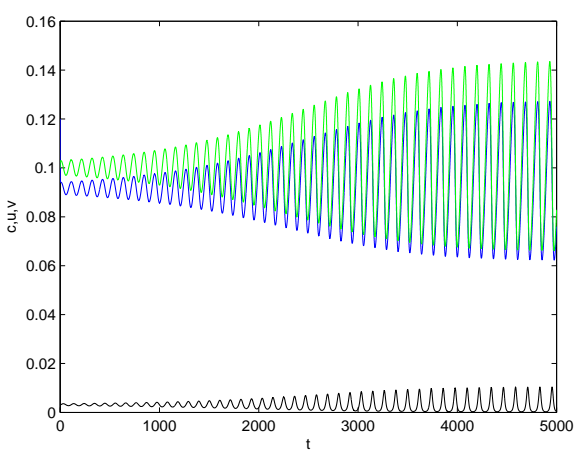

(b)

Figure 6 . Effect of changes in parameter $A$. The density of oxygen, phytoplankton and zooplankton versus time obtained for (a) $A=0.9, c_{1}=0.7$ and (b) $A=1, c_{1}=0.7$. In both cases, the initial conditions are $c_{0}=0.0916, u_{0}=0.1$ and $v_{0}=0.0031$. Other parameters are given in the text (see the first paragraph of Section 4). 


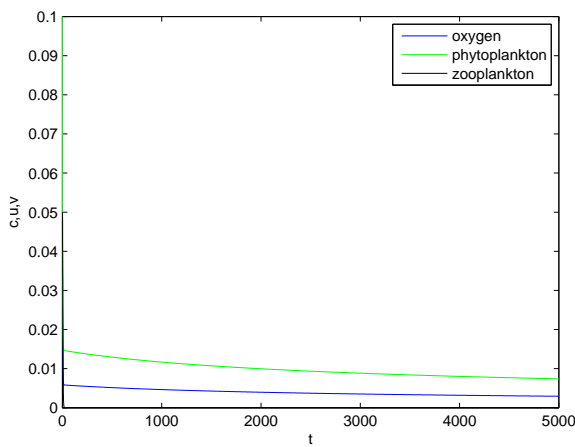

(a)

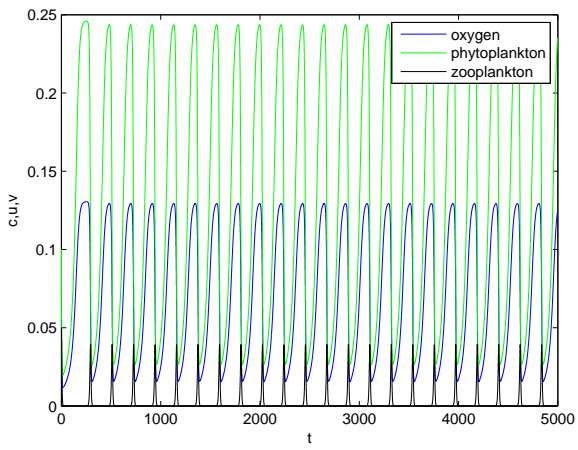

(c)

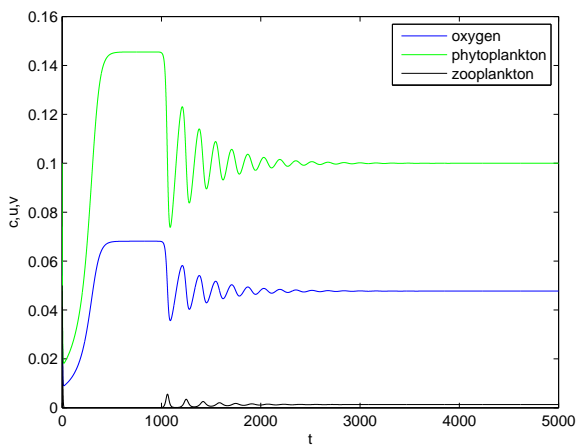

(b)

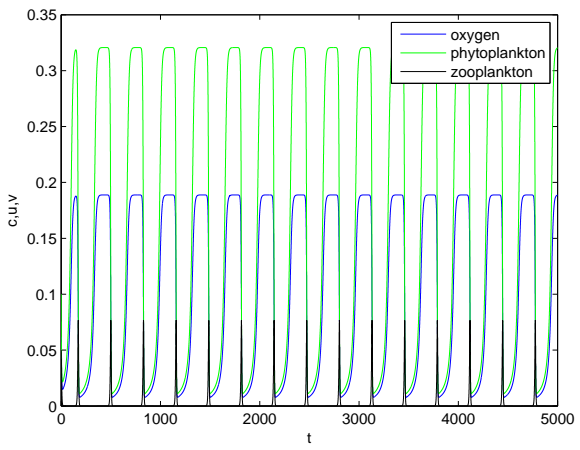

(d)

FiguRE 7. Effect of changes in parameter $A$. The density of oxygen, phytoplankton and zooplankton versus time obtained for (a) $A=0.41$, (b) $A=0.5$, (c) $A=0.6$ and (d) $A=0.7$. In all cases $c_{1}=0.4$, other parameters are the same as in the previous figures. The initial conditions are $c_{0}=0.06, u_{0}=0.1, v_{0}=0.05$.

The Hopf bifurcation occurs between $A=0.5$ and $A=0.6$, so that for $A=0.6$ (Fig. 7c) the system dynamics is periodical with the densities obviously following the stable limit cycle. A further increase in $A$ leads to an increase in the size of the limit cycle and to an increase in the period of oscillations; see Fig. 7d obtained for $A=0.7$.

In conclusion to this section, we mention that the system exhibit a similar succession of dynamical regimes in response to a change in parameter $c_{1}$; in particular, an increase in $c_{1}$ may result in the loss of stability of the coexistence state $E_{3}$ and the emergence of periodical oscillations. We do not show these simulations results here for the sake of brevity.

\section{Numerical simulations II: spatial dynamics}

Now we are going to consider the properties of the oxygen-plankton system in space. For this purpose, we consider a spatially explicit extension of the model (3.1-3.3) which is described by the following system 
of reaction-diffusion equations:

$$
\begin{aligned}
& \frac{\partial c}{\partial t}=D_{T} \frac{\partial^{2} c}{\partial x^{2}}+A\left(1-\frac{c}{c+1}\right) u-c, \\
& \frac{\partial u}{\partial t}=D_{T} \frac{\partial^{2} u}{\partial x^{2}}+\left(\frac{B c}{c+c_{1}}-u\right) u-\frac{u v}{u+h}, \\
& \frac{\partial v}{\partial t}=D_{T} \frac{\partial^{2} v}{\partial x^{2}}+\left(\frac{\beta u v}{u+h}\right)-\mu v .
\end{aligned}
$$

Here $c=c(x, t)$ is the concentrations of oxygen and $u=u(x, t)$ and $v=v(x, t)$ are the densities of phytoand zooplankton at time $\mathrm{t}$ and position $x$, and $D_{T}$ is the coefficient of the turbulent diffusion $[25,27]$.

We mention here that, in reality, the transport of oxygen in the sea water takes place due to the combined action of the molecular and turbulent diffusion, so that the coefficient in Eq. (5.1) should actually be $D_{T}+D_{0}$ rather than just $D_{T}$, where $D_{0}$ is the molecular diffusion coefficient. However, on the spatial scales relevant to the plankton dynamics (i.e. $10^{-1}$ to $10^{5}$ meters) the rate of molecular diffusion is known to be several orders of magnitude less than the that of the turbulent diffusion. Hence, $D_{0} \ll D_{T}$ and $D_{T}+D_{0} \approx D_{T}$. Similarly, the transport of zooplankton results from the interplay between the turbulent diffusion and the self-movement of the zooplankton organisms. However, the mixing due to the self-movement of zooplankton (which we assume to be random in space and described by the biodiffusion coefficient $D_{v}$ ) appears to be much less compared to the turbulent mixing [27], i.e. $D_{v} \ll D_{T}$, so that $D_{T}+D_{v} \approx D_{T}$.

We also mention here that, having considered appropriate scaling of the spatial coordinates as $x \rightarrow$ $x^{\prime}=x \sqrt{m / D_{T}}$, cf. the lines after Eqs. (2.5-2.6), the coefficient $D_{T}$ will disappear from the equations. Correspondingly, below we consider Eqs. (5.1-5.3) to be dimensionless by setting $D_{T}=1$. Also, we fix some of parameters $(\beta=1, \mu=0.5$ and $h=0.1)$ and focus on the effect of variations in $A$ and $c_{1}$.

Equations (5.1-5.3) are considered in a finite domain $0<x<L$ where parameter $L$ is the domain length. At the domain boundaries, the zero-flux boundary condition is imposed.

The choice of the initial conditions is a subtle issue as different initial conditions may result in very different spatiotemporal dynamics [21]. In this paper, we consider the initial species distribution describing a zooplankton patch in a space with uniformly distributed oxygen and phytoplankton:

$$
\begin{aligned}
& c(x, 0)=c_{0}, \\
& u(x, 0)=u_{0}, \\
& v(x, 0)=v_{0} \quad \text { for } \quad\left|x_{i}\right|<\epsilon, \quad \text { otherwise } \quad v(x, 0)=0,
\end{aligned}
$$

where $c_{0}, u_{0}$ and $v_{0}$ are thus the initial densities and $\epsilon$ is the patch diameter. The results shown below are obtained for $\epsilon=100$.

Equations (5.1-5.3) are solved numerically using the finite difference method. The mesh steps are chosen as $\triangle t=0.01$ and $\triangle x=0.5$ and it was checked that these values are sufficiently small to avoid any significant numerical artifacts.

Note that the insight into the properties of the nonspatial system that we made in Sections 2 and 3 creates a useful framework for the understanding of the properties of the spatiotemporal system (5.1-5.3). In particular, as we have observed in our numerical simulations (not shown here for the sake of brevity), if the conditions (3.8) for the existence of the positive steady state $E_{3}$ do not hold but the condition (2.20) for the existence of the zooplankton-free equilibrium $E_{2}$ hold, zooplankton eventually goes extinct over the whole space and the phytoplankton density $u$ and the oxygen concentration $c$ converge, in the course of time, to the spatially uniform distribution $c(x, t) \equiv \tilde{c}$ and $u(x, t) \equiv \tilde{u}$. In case the condition (2.20) does not hold either, then all three components eventually converge to zero everywhere in space.

However, we want to emphasize that the effect of space often appears to be difficult to predict basing on the properties of the nonspatial system only. In particular, it may happen that the species extinction 


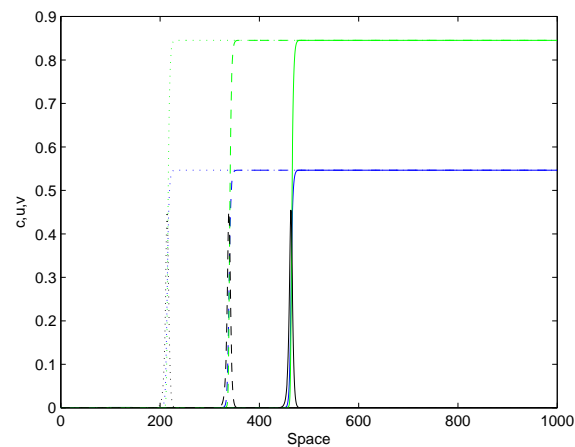

FiguRE 8. Distribution of oxygen (blue), phytoplankton (green) and zooplankton (black) over space at $t=100$ (dotted line), $t=200$ (dashed line) and $t=300$ (solid line) obtained for parameters $A=1$ and $c_{1}=0.1$ and the initial conditions (5.4-5.6), other parameters are given in the text.

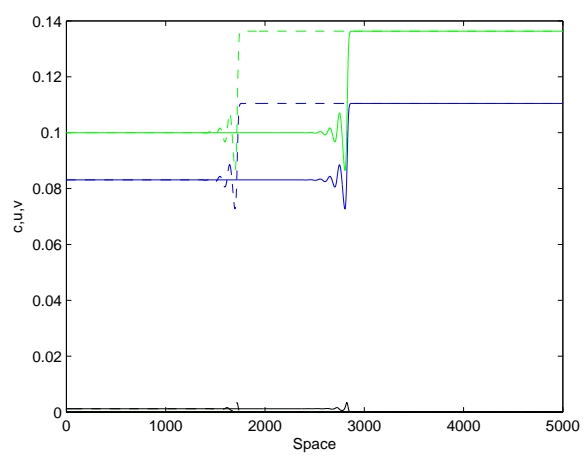

(a)

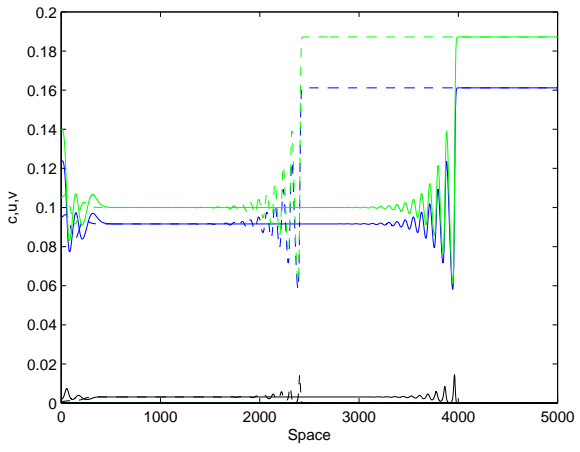

(b)

Figure 9. Snapshots of the density distribution over space for oxygen (blue), phytoplankton (green) and zooplankton (black) at $t=3000$ (dashed curves) and $t=5000$ (solid curves) obtained for (a) $A=0.9, c_{1}=0.7$ and (b) $A=1, c_{1}=0.7$. The initial conditions are $c_{0}=0.0916, u_{0}=0.1$ and $v_{0}=0.0031$.

can follow an interesting scenario. As an example, Fig. 8 shows the evolution of the species spatial distribution over time obtained for the same parameter values as Fig. 5. As it should be expected, in the large-time limit the system goes extinct; however, at an intermediate time, the initial distribution evolves to a travelling wave. The distribution of oxygen and phytoplankton forms a travelling front separating the area where these quantities are approximately at their steady state values $c(x, t)=\tilde{c}$ and $u(x, t)=\tilde{u}$ (on the right of the front) from the area where these quantities have gone extinct (on the left of the front). The zooplankton density forms a narrow peak at the position of the front. This solution of the model (5.1-5.3) apparently describes an interesting ecological situation. There is not enough oxygen anywhere in the system to support a stable existence of zooplankton; however, zooplankton can survive transiently (and over a relatively long time) at the interface between the area of partial depletion of oxygen and the area with no oxygen.

Figure 9 presents the simulation results in the spatial system (5.1-5.3) obtained for the parameters corresponding to Fig. 6. In particular, in Fig. 9a the coexistence state is a stable focus (the corresponding nonspatial dynamics is shown in Fig. 6a). It is readily seen that, in this case, the intermediate-time 
solution is given by an oscillating travelling front connecting the two steady states, i.e. the zooplanktonfree state $E_{2}$ (on the right of the front) and the coexistence state $E_{3}$ (on the left of the front). The front propagates to the right so that, in the large-time limit, the species densities converge to the spatially uniform distribution $c(x, t)=\bar{c}, u(x, t)=\bar{u}, v(x, t)=\bar{v}$.

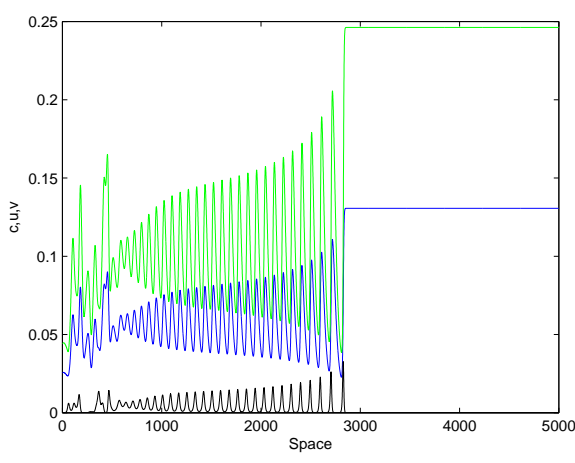

(a)

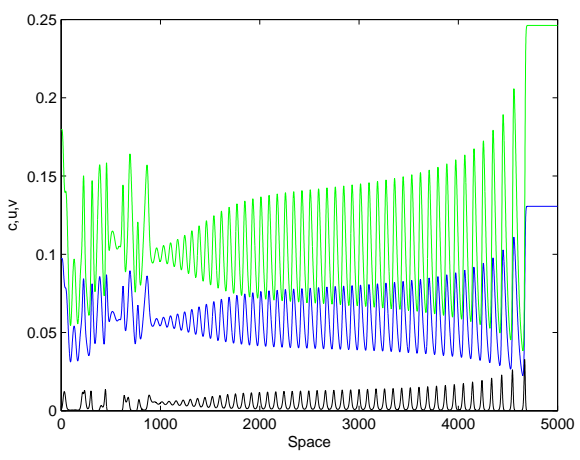

(b)

FiguRE 10. Distribution of oxygen (blue), phytoplankton (green) and zooplankton (black) over space at (a) $t=3000$ and (b) $t=5000$ obtained for $A=0.6$ and $c_{1}=0.4$ with the initial conditions $c_{0}=0.06, u_{0}=0.1$ and $v_{0}=0.05$; see $(5.4-5.6)$. Other parameters are given in the text, see the beginning of Section 5.

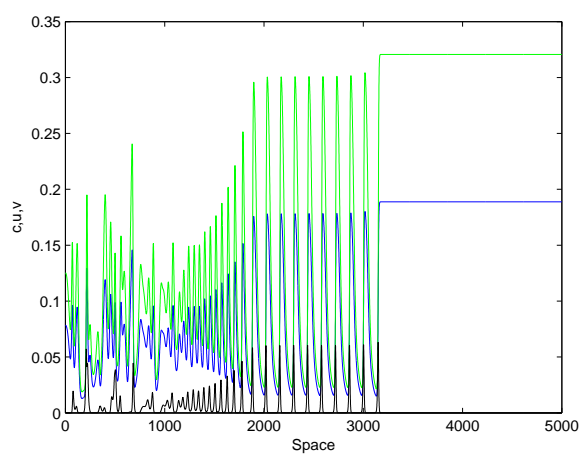

(a)

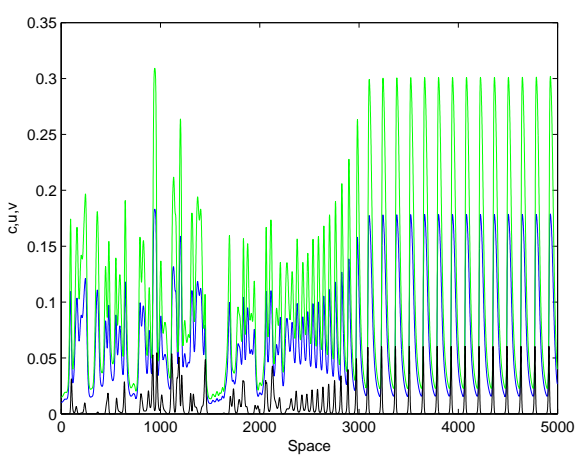

(b)

FiguRE 11. The density of oxygen (blue), phytoplankton (green) and zooplankton (black) over space at (a) $t=3000$ (b) $t=5000$ obtained for other given parameter values $A=0.7, c_{1}=0.4$ and initial conditions are the same for (a) and (b) as (5.4- 5.6) with $c_{0}=0.06, u_{0}=0.1$ and $v_{0}=0.05$.

In Fig. 9b, the parameters are beyond the Hopf bifurcation so that the nonspatial system becomes oscillatory (see Fig. 6b). In this case, the spatial species distribution has somewhat counter-intuitive properties. A part of the spatial solution forms, similarly to the above, an oscillating travelling front connecting $E_{2}$ on the right and $E_{3}$ on the left. However, the coexistence state $E_{3}$ is unstable and therefore cannot persist indefinitely. As the oscillating travelling front propagates to the right, far behind 
the front, i.e. behind the unstable plateau, irregular oscillations eventually develop; see the left-hand end of Fig. 6b. In the large-time limit, the irregular spatiotemporal oscillations eventually occupy the whole domain (not shown here). The system dynamics thus follow the generic scenario well-known for other reaction-diffusion systems, i.e., the onset of chaos in the wake of the front preceded by the so called dynamical stabilization of unstable equilibrium $[19,30,32,35]$.

The existence of travelling front connecting $E_{2}$ to $E_{3}$ is not a general property of the system (5.1-5.3) though. The unstable plateau does not always exists and, alternatively, the spatially uniform distribution of oxygen and phytoplankton (corresponding to the zooplankton-free state $E_{2}$ ) may give way to a band of regular spatial oscillations of varying amplitude; see Fig. 10 obtained for parameters where the nonspatial system is oscillatory. The band of regular spatial oscillations is eventually displaced by the chaotic spatiotemporal oscillations which, in the course of time, occupy the whole domain.

We mention here that the onset of spatiotemporal chaos in the system (5.1-5.3) is, in case the corresponding nonspatial system is oscillatory, a common property. A general tendency that we have observed in our simulations is that the spatial oscillations become more and more irregular with an increase in $A$ (keeping other parameters fixed), i.e. when the point in the parameter plane moves further away from the Hopf bifurcation curve. An example of this situation is shown in Fig. 11.

\section{Summary and concluding remarks}

Peculiarities of plankton dynamics in marine ecosystems have been a focus of significant interest and intense research for several decades. A considerable progress has been made in the understanding of factors and mechanisms underlying a variety of plankton phenomena, and there exist vast literature covering almost every aspect of the plankton research. However, there is at least one aspect that has been rather poorly investigated. Admittedly, plankton is not only the base of the ocean food chain (which is often mentioned as a practical reason justifying the effort behind scientific studies), it is also responsible for the production of about two thirds of the atmospheric oxygen. Surprisingly, there are very few studies directly concerned with the dynamics of the oxygen-plankton coupling. In spite of its obvious importance, this issue remains clearly overlooked both in theoretical and field studies.

In this paper, we developed a conceptual three-component mathematical model of the oxygen-phytozooplankton system. The model consists of three ordinary differential equations. We first considered the nonspatial version of the model which, in real-world terms, corresponds to a well-mixed system with spatially uniform distribution of species. The properties of the model have been studied both analytically and by simulations. In particular, we found analytical conditions for the existence of a (unique) positive equilibrium corresponding to the coexistence of all three components. In ecological terms, parameter values corresponding to the existence of the positive equilibrium may be regarded as safe, whilst the disappearance of this steady state should be regarded as an ecological disaster resulting in mass extinction of the plankton species.

We then considered a spatially explicit extension of our model which takes into account the transport of plankton and oxygen by the turbulent diffusion. The model is described by a system of three partial differential equations of reaction-diffusion type. The properties of the system were studied by extensive numerical simulations. We have shown that the model exhibits rich spatiotemporal dynamics, in particular, resulting in travelling fronts and spatiotemporal chaos. The observed properties of the system are therefore reminiscent of the dynamics of other ecologically relevant reaction-diffusion systems, e.g. see $[21,40]$, which helps to verify the model properties and to interpret the results.

Perhaps the most interesting property of our model is that phytoplankton is predicted to survive only if the rate of oxygen production is above a certain critical value; see condition (2.20). Since the rate of oxygen production may be expected to depend on the properties of the environment, it makes our model a convenient and relevant theoretical tool that can be used for the purposes of nature conservation and marine ecosystems management. This will become a focus of our future research. 


\section{Acknowledgements}

The authors are thankful to Nikolay Brilliantov (Leicester) for stimulating discussions at the early stage of this study. Andrew Morozov (Leicester) has read the manuscript and made a few helpful remarks aimed to improve the clarity of the text. Useful comments from two anonymous reviewers are appreciated.

\section{Appendix}

The linearized system's matrix is as follows:

$$
A=\left(\begin{array}{ccc}
-\frac{A u}{(1+c)^{2}}-1 ; & A\left(1-\frac{c}{1+c}\right) ; & 0 ; \\
\frac{B c_{1} u}{\left(c+c_{1}\right)^{2}} ; & \frac{B c}{c+c_{1}}-2 u-\frac{v h}{(u+h)^{2}} ; & -\frac{u}{u+h} ; \\
0 ; & \frac{\beta v h}{(u+h)^{2}} ; & \frac{\beta u}{u+h}-\mu ;
\end{array}\right)
$$

- Extinction steady state: $E_{1}=(0,0,0)$.

Matrix (6.1) takes the following form:

$$
\operatorname{det}\left(A_{(0,0,0)}-\lambda I\right)=\left|\begin{array}{rcc}
-1-\lambda ; & A ; & 0 ; \\
0 ; & -\lambda ; & 0 ; \\
0 ; & 0 ; & -\mu-\lambda ;
\end{array}\right|=0,
$$

so that o the characteristic equation is defined by

$$
\Rightarrow(-\mu-\lambda)(-\lambda)(-1-\lambda)=0 .
$$

From Eq. (6.2), $\lambda_{1}=-\mu, \lambda_{2}=0$ and $\lambda_{3}=-1$. Therefore, the origin has two negative real eigenvalues and one zero eigenvalue.

- Zooplankton-free steady state: $E_{2}=(\tilde{c}, \tilde{u}, 0)$.

The corresponding matrix is:

$$
\operatorname{det}\left(A_{(\tilde{c}, \tilde{u}, 0)}-\lambda I\right)=\left|\begin{array}{ccc}
-\frac{A \tilde{u}}{(1+\tilde{c})^{2}}-1-\lambda ; & A\left(1-\frac{\tilde{c}}{1+\tilde{c}}\right) ; & 0 ; \\
\frac{B c_{1} \tilde{u}}{\left(\tilde{c}+c_{1}\right)^{2}} ; & \frac{B \tilde{c}}{\tilde{c}+c_{1}}-2 \tilde{u}-\lambda ; & -\frac{\tilde{u}}{\tilde{u}+h} ; \\
0 ; & 0 ; & \frac{\beta \tilde{u}}{\tilde{u}+h}-\mu-\lambda ;
\end{array}\right|=0
$$

so that

$$
\begin{aligned}
\operatorname{det}\left(A_{(\tilde{c}, \tilde{u}, 0)}-\lambda I\right)= & {\left[\left(-\frac{A \tilde{u}}{(1+\tilde{c})^{2}}-1-\lambda\right)\left(\frac{B \tilde{c}}{\tilde{c}+c_{1}}-2 \tilde{u}-\lambda\right)\right.} \\
& \left.-\frac{A}{1+\tilde{c}} \frac{B c_{1} \tilde{u}}{\left(\tilde{c}+c_{1}\right)^{2}}\right]\left(\frac{\beta \tilde{u}}{\tilde{u}+h}-\mu-\lambda\right)=0,
\end{aligned}
$$

where $\tilde{u}$ and $\tilde{c}$ defined in Eqs.(2.11) and (2.13).

where

$$
\lambda_{1}=\frac{\beta \tilde{u}}{\tilde{u}+h}-\mu \quad \lambda_{2,3}=\frac{-\rho \pm \sqrt{\rho^{2}-4 \Delta}}{2}
$$

$$
\begin{gathered}
\rho=\frac{A \tilde{u}}{(1+\tilde{c})^{2}}-\frac{B \tilde{c}}{\tilde{c}+c_{1}}+2 \tilde{u}+1 \\
\Delta=\frac{2 A \tilde{u}^{2}}{(1+\tilde{c})^{2}}-\frac{A B \tilde{u} \tilde{c}}{(1+\tilde{c})^{2}\left(\tilde{c}+c_{1}\right)}-\frac{B \tilde{c}}{\tilde{c}+c_{1}}-\frac{A B c_{1} \tilde{u}}{(1+\tilde{c})\left(\tilde{c}+c_{1}\right)^{2}}+2 \tilde{u}
\end{gathered}
$$


- Coexistence steady state: $E_{3}=(\bar{c}, \bar{u}, \bar{v})$.

$$
\operatorname{det}\left(A_{(\bar{c}, \bar{u}, \bar{v})}-\lambda I\right)=\left|\begin{array}{ccc}
-\frac{A \bar{u}}{(1+\bar{c})^{2}}-1-\lambda ; & A\left(1-\frac{\bar{c}}{1+\bar{c}}\right) ; & 0 \\
\frac{B c_{1} \bar{u}}{\left(\bar{c}+c_{1}\right)^{2}} ; & \frac{B \bar{c}}{\bar{c}+c_{1}}-2 \bar{u}-\frac{\bar{v} h}{(\bar{u}+h)^{2}}-\lambda ; & -\frac{\bar{u}}{\bar{u}+h} ; \\
0 ; & \frac{\beta \bar{v} h}{(\bar{u}+h)^{2}} ; & \frac{\beta \bar{u}}{\bar{u}+h}-\mu-\lambda ;
\end{array}\right|=0
$$

so that

$$
\begin{aligned}
\operatorname{det}\left(A_{(\bar{c}, \bar{u}, \bar{v})}-\lambda I\right)= & \frac{\beta \bar{u} \bar{v} h}{(\bar{u}+h)^{3}}\left(-\frac{A \bar{u}}{(1+\bar{c})^{2}}-1-\lambda\right) \\
& +\left(\frac{\beta \bar{u}}{\bar{u}+h}-\mu-\lambda\right)\left[( - \frac { A \overline { u } } { ( 1 + \overline { c } ) ^ { 2 } } - 1 - \lambda ) \left(\frac{B \bar{c}}{\bar{c}+c_{1}}-2 \bar{u}\right.\right. \\
& \left.\left.\quad-\frac{\bar{v} h}{(\bar{u}+h)^{2}}-\lambda\right)-\frac{A}{1+\bar{c}} \cdot \frac{B c_{1} \bar{u}}{\left(\bar{c}+c_{1}\right)^{2}}\right]=0,
\end{aligned}
$$

where $\bar{c}, \bar{u}$ and $\bar{v}$ defined by Eqs. (3.7).

Let

$$
\begin{gathered}
\phi=\frac{\beta \bar{u}}{\bar{u}+h}-\mu-\frac{A \bar{u}}{(1+\bar{c})^{2}}-1+\frac{B \bar{c}}{\bar{c}+c_{1}}-2 \bar{u}-\frac{\bar{v} h}{(\bar{u}+h)^{2}}, \\
\varepsilon=\frac{A \beta \bar{u}^{2}}{(1+\bar{c})^{2}(\bar{u}+h)}+\frac{\beta \bar{u}}{\bar{u}+h}-\frac{\beta B \bar{u} \bar{c}}{\left(\bar{c}+c_{1}\right)(\bar{u}+h)}+\frac{2 \bar{u}^{2} \beta}{\bar{u}+h}-\frac{A \bar{u} \mu}{(1+\bar{c})^{2}}-\mu \\
+\frac{B \bar{c} \mu}{\bar{c}+c_{1}}-2 \bar{u} \mu-\frac{\bar{v} h \mu}{(\bar{u}+h)^{2}}+\frac{A B \bar{u} \bar{c}}{(1+\bar{c})^{2}\left(\bar{c}+c_{1}\right)}-\frac{2 A \bar{u}^{2}}{(1+\bar{c})^{2}}-\frac{A \bar{u} \bar{v} h}{(1+\bar{c})^{2}(\bar{u}+h)^{2}} \\
+\frac{B \bar{c}}{\bar{c}+c_{1}}-2 \bar{u}-\frac{\bar{v} h}{(\bar{u}+h)^{2}}+\frac{A B \bar{u} c_{1}}{(1+\bar{c})\left(\bar{c}+c_{1}\right)^{2}},
\end{gathered}
$$

and

$$
\begin{aligned}
\rho= & -\frac{A \beta \bar{u}^{2} \bar{v} h}{(\bar{u}+h)^{3}(1+\bar{c})^{2}}-\frac{\beta \bar{u} \bar{v} h}{(\bar{u}+h)^{3}}-\frac{A B \beta \bar{u}^{2} \bar{c}}{(1+\bar{c})^{2}\left(\bar{c}+c_{1}\right)(\bar{u}+h)}+\frac{2 A \bar{u}^{3} \beta}{(1+\bar{c})^{2}(\bar{u}+h)} \\
& +\frac{A \beta \bar{u}^{2} \bar{v} h}{(1+\bar{c})^{2}(\bar{u}+h)^{3}}-\frac{B \beta \bar{u} \bar{c}}{\left(\bar{c}+c_{1}\right)(\bar{u}+h)}+\frac{2 \beta \bar{u}^{2}}{\bar{u}+h}+\frac{\beta \bar{u} \bar{v} h}{(\bar{u}+h)^{3}} \\
& -\frac{A B \beta \bar{u}^{2} c_{1}}{(1+\bar{c})\left(\bar{c}+c_{1}\right)^{2}(\bar{u}+h)}+\frac{A B \bar{u} \bar{c} \mu}{(1+\bar{c})^{2}\left(\bar{c}+c_{1}\right)}-\frac{2 A \bar{u}^{2} \mu}{(1+\bar{c})^{2}}-\frac{A \bar{u} \bar{v} h \mu}{(1+\bar{c})^{2}(\bar{u}+h)^{2}} \\
& +\frac{B \bar{c} \mu}{\bar{c}+c_{1}}-2 \bar{u} \mu-\frac{\bar{v} h \mu}{(\bar{u}+h)^{2}}+\frac{A B c_{1} \bar{u} \mu}{(1+\bar{c})\left(\bar{c}+c_{1}\right)^{2}} .
\end{aligned}
$$

Then the eigenvalues of system (6.7) are the solutions of the following cubic equation:

$$
\lambda^{3}-\phi \lambda^{2}-\varepsilon \lambda-\rho=0,
$$

The roots of equation (6.8) are:

$$
\begin{aligned}
\lambda_{1}=\frac{\phi}{3}+ & \frac{2^{\frac{1}{3}}\left(-\phi^{2}-3 \varepsilon\right)}{3\left(-27 \rho-2 \phi^{3}-9 \phi \varepsilon+3 \sqrt{3} \sqrt{27 \rho^{2}+4 \rho \phi^{3}+18 \rho \phi \varepsilon-\phi^{2} \varepsilon^{2}-4 \varepsilon^{3}}\right)^{\frac{1}{3}}} \\
& -\frac{\left(-27 \rho-2 \phi^{3}-9 \phi \epsilon+3 \sqrt{3} \sqrt{27 \rho^{2}+4 \rho \phi^{3}+18 \rho \phi \varepsilon-\phi^{2} \varepsilon^{2}-4 \varepsilon^{3}}\right)^{\frac{1}{3}}}{3 \cdot 2^{\frac{1}{3}}}
\end{aligned}
$$




$$
\begin{aligned}
\lambda_{2}= & \frac{\phi}{3}-\frac{(1+i \sqrt{3})\left(-\phi^{2}-3 \varepsilon\right)}{3 \cdot 2^{\frac{2}{3}}\left(-27 \rho-2 \phi^{3}-9 \phi \varepsilon+3 \sqrt{3} \sqrt{27 \rho^{2}+4 \rho \phi^{3}+18 \rho \phi \varepsilon-\phi^{2} \varepsilon^{2}-4 \varepsilon^{3}}\right)^{\frac{1}{3}}} \\
& +\frac{(1-i \sqrt{3})\left(-27 \rho-2 \phi^{3}-9 \phi \varepsilon+3 \sqrt{3} \sqrt{27 \rho^{2}+4 \rho \phi^{3}+18 \rho \phi \varepsilon-\phi^{2} \varepsilon^{2}-4 \varepsilon^{3}}\right)^{\frac{1}{3}}}{6 \cdot 2^{\frac{1}{3}}} \\
\lambda_{3}= & \frac{\phi}{3}-\frac{(1-i \sqrt{3})\left(-\phi^{2}-3 \varepsilon\right)}{3 \cdot 2^{\frac{2}{3}}\left(-27 \rho-2 \phi^{3}-9 \phi \varepsilon+3 \sqrt{3} \sqrt{27 \rho^{2}+4 \rho \phi^{3}+18 \rho \phi \varepsilon-\phi^{2} \varepsilon^{2}-4 \varepsilon^{3}}\right)^{\frac{1}{3}}} \\
& +\frac{(1+i \sqrt{3})\left(-27 \rho-2 \phi^{3}-9 \phi \varepsilon+3 \sqrt{3} \sqrt{27 \rho^{2}+4 \rho \phi^{3}+18 \rho \phi \varepsilon-\phi^{2} \varepsilon^{2}-4 \varepsilon^{3}}\right)^{\frac{1}{3}}}{6 \cdot 2^{\frac{1}{3}}}
\end{aligned}
$$

Therefore, the equilibrium $(\bar{c}, \bar{u}, \bar{v})$ has one real and two complex eigenvalues; hence, it is a mixed focus-type equilibrium.

\section{References}

[1] M. Abbott. Phytoplankton patchiness: ecological implications and observation methods. In: Patch dynamics (Levin, S.A., Powell, T.M., Steele, J.H., eds.), Lecture Notes in Biomathematics vol. 96. Springer, Berlin, 1993, pp. 37-49.

[2] W. Allegretto, C. Mocenni, A. Vicino. Periodic solutions in modelling lagoon ecological interactions. J. Math. Biol., 51 (2005), 367-388.

[3] M. Banerjee, S.V. Petrovskii. Self-organised spatial patterns and chaos in a ratio-dependent predator-prey system. Theoretical Ecology, 4 (2011), 37-53.

[4] M. Bengfort, U. Feudel, F.M. Hilker, H. Malchow. Plankton blooms and patchiness generated by heterogeneous physical environments. Ecological Complexity, 20 (2014), 185-194.

[5] J. Chattopadhyay, S. Pal. Viral infection on phytoplankton zooplankton system a mathematical model. Ecological Modelling, 151 (2002), 15-28.

[6] L. Edelstein-Keshet. Mathematical models in biology. Random House, New York, 1988.

[7] A.M. Edwards, J. Brindley. Zooplankton mortality and the dynamical behavior of plankton population models. Bull. Math. Biol., 61 (1999), 303-339.

[8] M.J.R. Fasham. The statistical and mathematical analysis of plankton patchiness. Oceanogr. Mar. Biol. Annu. Rev., 16 (1978), 43-79.

[9] T. Gaarder, H.H. Gran. Investigation of the production of plankton in the Oslo fjord, Rapp. et Proc-Verb. Cons. Int. Explor. Mer., 42 (1927), 3-48.

[10] M. Guysinsky, B. Hasselblatt, V. Rayskin. Differentiability of the Hartman-Grobman Linearization. Discr. Contin. Dyn. Syst., 9 (2003), 979-984.

[11] G.P. Harris. Phytoplankton ecology. Structure function and fluctuation. Chapman and Hall, USA, 1986.

[12] V. Hulla, L. Parrell, M. Falcucci. Modelling dissolved oxygen dynamics in coastal lagoons. Ecol. Model., 211 (2008), 468-480.

[13] Z. Jin, T.P. Charlock, W.L. Smith Jr., K. Rutledge. A parametrization of ocean surface albedo. Geophys. Res. Lett., 31 (2004), L22301, doi:10.1029/2004GL021180.

[14] Y.A. Kuznetsov. Elements of applied bifurcation theory. Applied Mathematical Sciences vol. 112. Springer, Berlin, 1995.

[15] T.K. Leen. A Coordinate Independent Center Manifold Reduction. Phys. Lett., 174 (1993), 89-93.

[16] N.D Lewis, A. Morozov, M.N. Breckels, M. Steinke, E.A. Codling. Multitrophic interactions in the sea: assessing the effect of infochemical-mediated foraging in a 1-d spatial model. Math. Model. Nat. Phenom., 8(6) (2015), 25-44.

[17] F. Mackas, C.M. Boyd. Spectral analysis of zooplankton spatial heterogeneity. Science, 204 (1979), 62-64.

[18] H. Malchow. Spatio-temporal pattern formation in nonlinear nonequilibrium plankton dynamics. Proc. R. Soc. Lond. B., 251 (1993), 103-109.

[19] H. Malchow, S.V. Petrovskii. Dynamical stabilization of an unstable equilibrium in chemical and biological systems. Mathematical and Computer Modelling, 36 (2002), 307-319.

[20] H. Malchow, S.V. Petrovskii, F. Hilker. Models of spatiotemporal pattern formation in plankton dynamics. Nova Acta Leopoldina, 88 (2003), 325-340.

[21] H. Malchow, S.V. Petrovskii, E. Venturino. Spatiotemporal patterns in ecology and epidemiology: theory, models, simulations. Mathematical and Computational Biology Series. Chapman and Hall / CRC Press, Boca Raton, 2008.

[22] N. Marchettini, C. Mocenni, A. Vicino. Integrating slow and fast dynamics in a shallow water coastal lagoon. Annali di Chimica, 89 (1999), 505-514.

[23] A.K. Misra. Modeling the depletion of dissolved oxygen in a lake due to submerged macrophytes. Nonlinear Analysis: Modeling and Control, 15 (2010), 185-198. 
[24] C. Mocenni. Mathematical modelling of coastal systems: engineering approaches for parameter identification, validation and analysis of the models. Unpublished manuscript, (2006), available at http : //web.univ - ubs.fr/lmam/frenod/SiteGdmEtGdm2/GGDDMM/03D22BA4 - $6225 \quad-46 C C \quad-8968-$ D8621FBFD $967_{f}$ iles/conf Mocenni.pdf.

[25] A.S. Monin, A.M. Yaglom. Statistical fluid mechanics, Vol. 1. MIT Press, Cambridge MA, 1971.

[26] B. Moss. Ecology of Freshwaters, 4th edition. John Willey, Chichester, 2010.

[27] A. Okubo. Diffusion and ecological problems: mathematical models. Springer-Verlag, Berlin, 1980.

[28] E. Paasche. Pelagic production in nearshore waters. In: Nitrogen cycling in coastal marine environments (Blackburn H., Sorensen J., eds.), John Wiley, Chichester, 1988, 33-58.

[29] M. Pascual. Diffusion-induced chaos in a spatial predator-prey system. Proc. R. Soc. Lond. B., 251 (1993), 1-7.

[30] S.V. Petrovskii, H. Malchow. Critical phenomena in plankton communities: KISS model revisited. Nonlinear Analysis: Real World Applications, 1 (2000), 37-51.

[31] S.V. Petrovskii, H. Malchow. Mathematical models of marine ecosystems. In: The Encyclopedia of Life Support Systems (EOLSS). Developed under the Auspices of the UNESCO. Eolss Publishers, Oxford, 2005. [http://www.eolss.net]

[32] S.V. Petrovskii, K. Kawasaki, F. Takasu, N. Shigesada. Diffusive waves, dynamical stabilization and spatio-temporal chaos in a community of three competitive species. Japan Journal of Industrial and Applied Mathematics, 18 (2001), 459-481.

[33] S. Rinaldi, R. Soncini-sessa, H. Stehfest, H. Tamura. Modeling and control of river quality. McGraw-Hill, 1979.

[34] L.A. Segel, J.L. Jackson. Dissipative structure: an explanation and an ecological example. J. Theor. Biol., 37 (1972), 545-559.

[35] J.A. Sherratt, M.A. Lewis, A.C. Fowler. Ecological chaos in the wake of invasion. Proc. Natl. Acad. Sci. USA, 92 (1995), 2524-2528.

[36] I.R. Smith. A simple theory of algal deposition, Freshwater Biol., 12 (1982), 445-449.

[37] J.H. Steele. Spatial pattern in plankton communities, Plenum Press, New York, 1978.

[38] J.H. Steele, E.W. Henderson. A simple plankton model. Am. Nat., 117 (1981), 676-691.

[39] A.A. Voinov, A.P. Tonkikh. Qualitative model of eutrophication in macrophyte lakes. Ecol. Model., 35 (1987), $211-226$.

[40] V. Volpert, S. Petrovskii. Reaction-diffusion waves in biology. Phys. Life Rev., 6 (2009), 267-310.

[41] M. Warkentin, H.M. Freese, U. Karsten, R. Schumann. New and fast method to quantify respiration rates of bacterial and plankton communities in freshwater ecosystem by using Optical Oxygen Sensor Spots. Applied and Environmental Microbiology, 73 (2007), 6722-6729. 